\title{
Practical use of the Modified Bronnikov Algorithm in micro-CT
}

\author{
M. Boone ${ }^{\mathrm{a}, *}$, Y. De Witte ${ }^{\mathrm{a}}$, M. Dierick ${ }^{\mathrm{a}}$, J. Van den Bulcke ${ }^{\mathrm{b}}$, J. Vlassenbroeck ${ }^{\mathrm{a}}$, L. Van Hoorebeke ${ }^{\mathrm{a}}$ \\ ${ }^{a}$ Ghent University, Subatomic and Radiation Physics \\ Proeftuinstraat 869000 Gent \\ ${ }^{b}$ Ghent University, Department of Forest and water management \\ Coupure Links 6539000 Gent
}

\begin{abstract}
Through the years, the resolution of X-ray Computed Tomography (CT) systems has increased rapidly, in particular for the newer micro- and nano-CT systems. With this increasing resolution, the limits of absorption contrast CT are being reached. At the same time, a new type of contrast becomes visible: phase-contrast. Mainly for low absorbing objects such as insects and wood, phase-contrast can lead to a new type of CT reconstruction using the Modified Bronnikov Algorithm (MBA) 1]. Despite it's theoretical limitation to pure phase objects, the algorithm has some clear advantages with respect to filtered back-projection (FBP). The MBA is therefore commonly used at the Centre for X-ray Tomography of the Ghent University (UGCT) to obtain additional information for optimal scanning results.
\end{abstract}

Full article can be found at http://dx.doi.org/10.1016/j.nimb.2009.01.129

Key words: Phase contrast, Modified Bronnikov Algorithm, Phase propagation imaging, micro-CT PACS: 42.30.Wb, 81.70.Tx

\section{Introduction}

Since the development of X-ray computed tomography (CT) by Hounsfield in 1972, the basic principle has not changed. A sample is placed between an X-ray source and a detector and rotated relative to the source-detector system. X-rays are attenuated in the sample, giving rise to a projection image. By taking images at different angles, the linear attenuation coefficient $\mu$ can be calculated by the standard filtered back projection algorithm (FBP) in each volume element of the object(voxel), starting from the Lambert-Beer law

$$
I / I_{0}=\int_{X-\text { raypath }} e^{-\mu} \mathrm{d} L
$$

where $I_{0}$ is the incident X-ray intensity and $I$ the transmitted intensity. [2]

For low-density objects such as wood, $\mu$ is rather low. For increasing resolution, thus when samples become very small, this leads to insufficient absorption contrast for a good reconstruction. At the same time, a new type of contrast becomes visible for this kind of samples: phase contrast.

Caused by a phase shift of the propagating wave, a typical edge enhancement effect is visible in the images. Although this results in seemingly sharpened radiographs, it induces

\footnotetext{
*Corresponding author. Tel. +32 92646628 Fax +329264 6697 Email addresses: Matthieu.Boone@UGent.be (M. Boone) $U R L:$ http://www.ugct.ugent.be (M. Boone)
}

erroneous values and artefacts in the CT reconstruction. Different techniques are available to exploit this effect. For $\mathrm{x}$-ray tubes the two most popular are grating interferometry [3, 4] and phase propagation imaging [5, 6]. Other techniques are available at synchrotron radiation setups, but considering the high operating cost and low accessibility, these do not serve the purpose of easy and frequent usage.

Phase propagation imaging results in mixed phase and absorption images. A reconstruction algorithm to reconstruct only the phase component of these images, the Modified Bronnikov Algorithm (MBA), will be explained in this article and tested on several different samples. The advantages and disadvantages of the algorithm will be shown on the basis of these examples.

\section{Theoretical background}

The interactions of light with a medium can be described by its refractive index $n(\lambda)$ :

$$
n(\lambda)=1-\delta(\lambda)+i \beta(\lambda)
$$

where $\lambda$ is the wavelength. The imaginary part $i \beta$ is the extinction coefficient, responsible for absorption of the wave in the medium. The real part $1-\delta$ denotes the ratio of the phase velocity of the wave through vacuum and the phase velocity in the medium. For visible light, this $\delta$ is between 0 and -1 for most materials, meaning the phase velocity is smaller in a medium than it is in vacuum, which causes refraction, a deviation of the propagation direction of the 
light. For x-rays, the absolute value of $\delta$ is much lower, in the order of $+10^{-6}$. This means the deviations are very small and only visible at interfaces that are almost parallel to the X-rays. Another phenomenon caused by phase shift, diffraction, can be disregarded at conventional microCT scanners using an x-ray tube, because the coherence length $L_{T}[7$

$$
L_{T}=\lambda S / D
$$

where $S$ is the spot size of the source and $D$ is the distance from the source to the object, is smaller than the resolution at these setups.

It can be proven that for objects with small variations in absorption, the intensity at the detector is given by [8, 9, 10]

$$
I_{\theta}^{d}(x, y)=I_{\theta}^{d=0}(x, y)\left(1-\frac{d \lambda}{2 \pi} \nabla^{2} \phi_{\theta}(x, y)\right)
$$

where $x, y$ are the coordinates on the detector plane, $d$ is the distance from object to detector, $\theta$ is the projection angle and $\phi$ the phase function:

$$
\phi_{\theta}(x, y)=\frac{2 \pi}{\lambda} \int_{\text {raypath }} \delta\left(x^{\prime}, y^{\prime}, z^{\prime}\right) \mathrm{d} x^{\prime} \mathrm{d} y^{\prime} \mathrm{d} z^{\prime}
$$

Eq. (4) is known as the Transport of Intensity Equation (TIE) and is a commonly used approximation for simulations 11, 12.

Defining the data function

$$
g_{\theta}(x, y)=I_{\theta}^{d}(x, y) / I_{\theta}^{d=0}(x, y)-1
$$

a reconstruction algorithm similar to the FBP is derived by Bronnikov [13, 14], by which the coefficient $\delta$ is calculated by:

$$
\delta\left(x^{\prime}, y^{\prime}, z^{\prime}\right)=\frac{1}{4 \pi^{2} d} \int_{0}^{\pi} q * * g_{\theta} \mathrm{d} \theta
$$

where the filter function

$$
q(x, y)=\frac{|y|}{x^{2}+y^{2}}
$$

is convolved with the data function $g_{\theta}$. This convolution can easily be implemented in the Fourier domain where the filter has the following form:

$$
Q(\xi, \eta)=\frac{|\xi|}{\xi^{2}+\eta^{2}}
$$

In the numerator of this filter, one can recognize the ramp filter $|\xi|$ of standard FBP. For easy implementation, the filtering is done separately where only the denominator is retained, because the ramp filter is implemented in the FBP reconstruction software. This reduces the algorithm to one extra filtering step in the reconstruction progress. In this algorithm, it was assumed that $g_{\theta}$ is known. For a conical beam setup, $I_{\theta}^{d} / I_{\theta}^{d=0}$ can not be calculated because of the changing magnification, except for objects with no absorption, i.e. pure phase objects. For low-absorbing objects, this problem can be partially corrected by adding a correction parameter to the filter

$$
Q_{c o r}(\xi, \eta)=\frac{|\xi|}{\xi^{2}+\eta^{2}+\alpha}
$$

as suggested by Groso et al. [15, who named this algorithm the Modified Bronnikov Algorithm (MBA). A similar algorithm, the TIE phase retrieval, was derived by Paganin et al. [16] and successfully applied by Mayo et al. [17. When the assumption of a homogeneous object is made, the presence of the parameter $\alpha$ can be derived directly from the TIE. The value of this parameter is determined using a semi empirical approach. A too small constant leads to blurry results, where a too large constant eliminates the filter. Based on a single filtered projection, a good compromise can easily be chosen for the best results.

\section{Experimental details}

One of the main advantages of the MBA is that there is no extra equipment required. The setup can be any microCT scanner where the detector pixel-size is small enough to observe the very low refraction angles. At the micro-CT scanner of UGCT [18, a resolution down to $1 \mu \mathrm{m}$ can be achieved. The setup consists mainly of a Feinfocus nanofocus X-ray source and different X-ray detectors. The tube can reach a spotsize of $1 \mu \mathrm{m}$ at voltages up to $60 \mathrm{kV}$. Two different detectors, a Varian PaxScan 2520V and a Photonic Science VHR, were used. The first has $1880 \times 1496$ pixels with a pixelsize of $127 \times 127 \mu \mathrm{m}^{2}$, while the Photonic Science has a high resolution, with $2667 \times 4008$ pixels of $9 \mu \mathrm{m} \times 9 \mu \mathrm{m}$. Depending on the desired resolution and thus the used detector, the X-ray source energy was chosen between 60 and $80 \mathrm{keV}$. No hardware filtering was applied. Total scan time was approximately 2 hours for all samples. Samples were gathered in different research domains, to illustrate the practical use of the MBA for fast and low-cost phase contrast imaging. Some of these samples are also interesting for industrial purposes, which makes the algorithm useful outside the field of fundamental research.

1. A wood sample with fungus inside. The fungus fills some pores, giving an indication of the penetration of the fungus. The sample is taken at the edge of a tree ring, resulting in different wall thicknesses in the sample.

2. An optical fibre, an industrial application.

3. A dried head louse. More generally, the MBA is useful for all kinds of small insects.

4. A pharmaceutical sample. Micro-CT is of great interest for pharmacy because it can give valuable information on porosity and internal formation. 
All reconstructions were performed by the tomographic reconstruction software Octopus, which allows to perform the processing steps individually [19]. Rendering was done with Volume Graphics VGStudioMax 1.2.1.

\section{Results and discussion}

\subsection{Wood sample}

The main scientific interest in the wood sample are the pores filled with fungus. When the grayscale is clipped at 0 , which is physically equivalent to ignoring negative densities, the fungus is almost invisible in the FBP reconstruction. These features become visible when reconstructed with MBA (Figure 1). As stated by Bronnikov, the algorithm contains a low-pass filter, making it very stable for high-frequency noise. This is in contrast with the FBP filter, where high-frequency noise is enhanced by the ramp filter. This explains the lower noise in the MBA-reconstructed images. A drawback of this noise stability is the smoothing of small details. If the fungus has an internal structure, it is not visible on this MBA reconstruction. The FBP reconstruction is not conclusive on this matter either because of the high noise. Visual microscopy revealed the internal structure was below the resolution of the CT-scan, while homogeneity on the scale of the obtained resolution was hard to evaluate. Although this blurring is a great drawback, the MBA made the fungus visible and made it possible to segment the volume, which was the primary goal.

\subsection{Optical fibre}

The optical fibre is a very well-known sample which makes verification of the results easy. The fibre exists of a dense core with a less dense cladding. These two are comprised in another cladding layer. Both cladding layers are perfectly homogeneous, thus any dense edge is known to be a false result. These artefacts are indeed visible on the FBP reconstruction, but are successfully removed by the MBA (Figure 2), indicating the correct functioning of the algorithm.

At the same time, this sample shows clearly the great drawback of the MBA. The reconstruction has become very blur, and small details are no longer visible. The incision disappears too fast in line profiles, and has higher gray values than the inner cladding. This same disadvantage is visible in all the other samples, yet it is most obvious in this sample.

\subsection{Head louse}

A very interesting field to use of the MBA is biology. Small animals such as insects and spiders are lowabsorbing samples, with high detail inside. These samples give a lot of information on the advantages and drawbacks of the MBA compared to standard FBP. Besides the slices, which show the same removal of artificial edges and streaks and the blurring of the reconstruction, the 3D renderings are of great interest for this kind of samples, showing an overview of the sample.

The streaks and artificial edges in the FBP reconstruction result in structural noise around the sample and badly visible structure inside the sample. The MBA rendering is much smoother, making structure inside the sample more distinct (Figure 3). Some details such as hairs are again lost due to the blurring effect of the filter.

\subsection{Pharmaceutical sample}

The pharmaceutical sample is a mini-tablet of about $3 \mathrm{~mm}$ diameter, degraded by demineralised water. It is made of a light material where phase contrast results in false densities at the border and streaks in the interiour caused by the pores when reconstructed with FBP. Since porosity is of great interest in this kind of samples, a good thresholding is of great importance. This thresholding is disturbed by the streaks and other artefacts in the FBP reconstruction. By using $\mathrm{MBA}$, the interior of the sample is more uniform, making porosity measurements much more reliable. This becomes clear in Fig, 4 . This difference is clearly visible on line profiles. Again, some details are lost in the MBA reconstruction. However, most details that seem to be lost are not representative due to their very small size (less than 3 voxels)

\section{Conclusion}

It is shown in this article that the Modified Bronnikov Algorithm can succesfully reconstruct the distribution of $\delta$ by application of the filter (9) for numerous samples, analogeous to the reconstruction of the attenuation parameter $\mu$ in conventional $\mathrm{CT}$. This algorithm can be used at any micro-CT setup where phase contrast is present in the images, making it an excellent low-cost phase reconstruction algorithm. Although an inconvenient blurring is present in the MBA-reconstructed images, they comprise valuable additional information about the samples besides the information gathered with FBP. It is therefore useful for some samples to do both reconstructions in order to obtain as much information as possible.

\section{Acknowledgements}

The authors are very grateful to Ellen Verhoeven of the Laboratory of Pharmaceutical Technology (UGent) and Kris Honraet from Oystershell NV for their cooperation and permission to use their samples. The Special Research Fund of the Ghent University (BOF) is acknowledged for the doctoral grant to Y. De Witte, the post-doc grant to M. Dierick and for their financial support.

\section{References}

[1] A. V. Bronnikov, Theory of quantitative phase-contrast computed tomography, J. Opt. Soc. Am. A 19 (3) (2002) 472-480. 

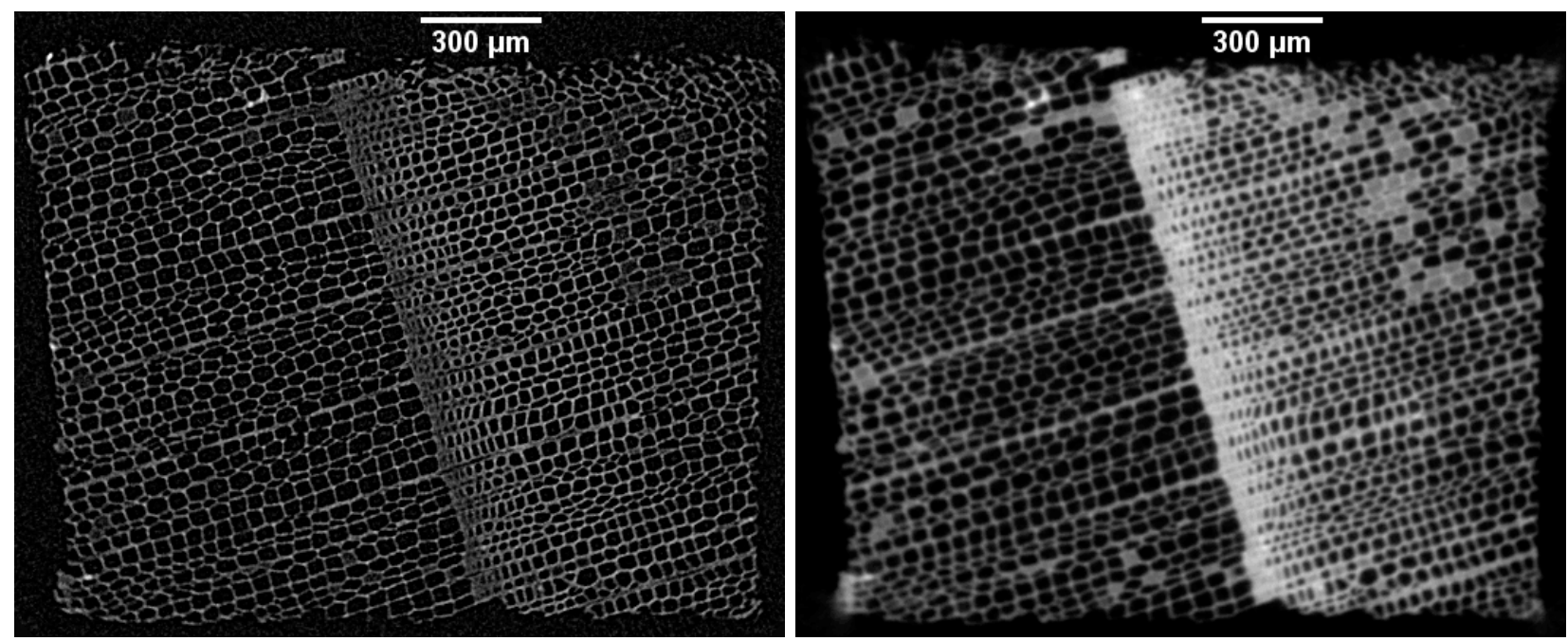

Figure 1: Reconstructed slice from a wood sample with fungus inside. left: standard FBP, right: MBA
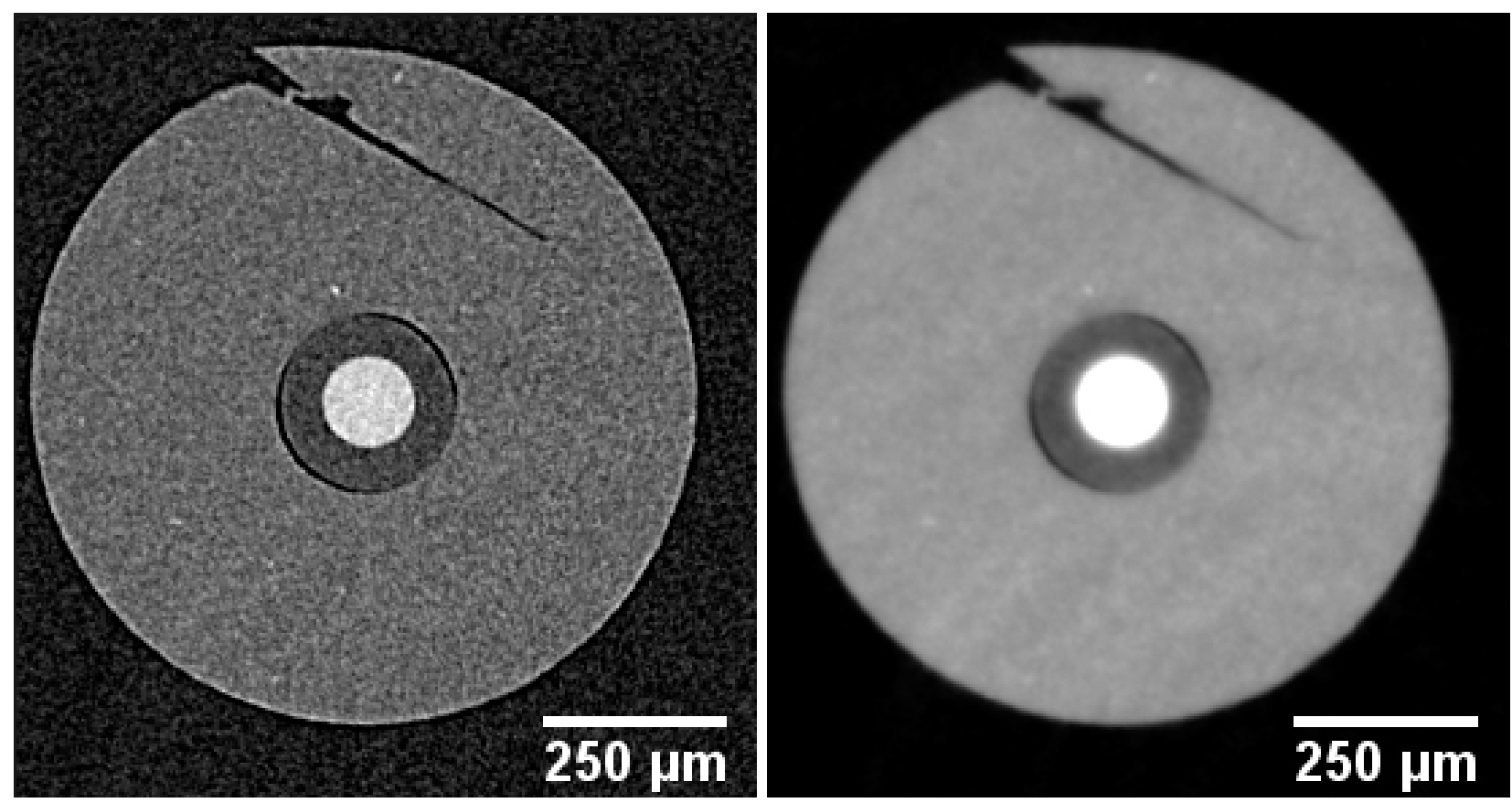

Figure 2: Reconstructed slice from an optical fibre. The artificial edges are clearly visible on the FBP-reconstructed image (left). These are absent in the MBA-reconstructed image, which is blurrier (right) 


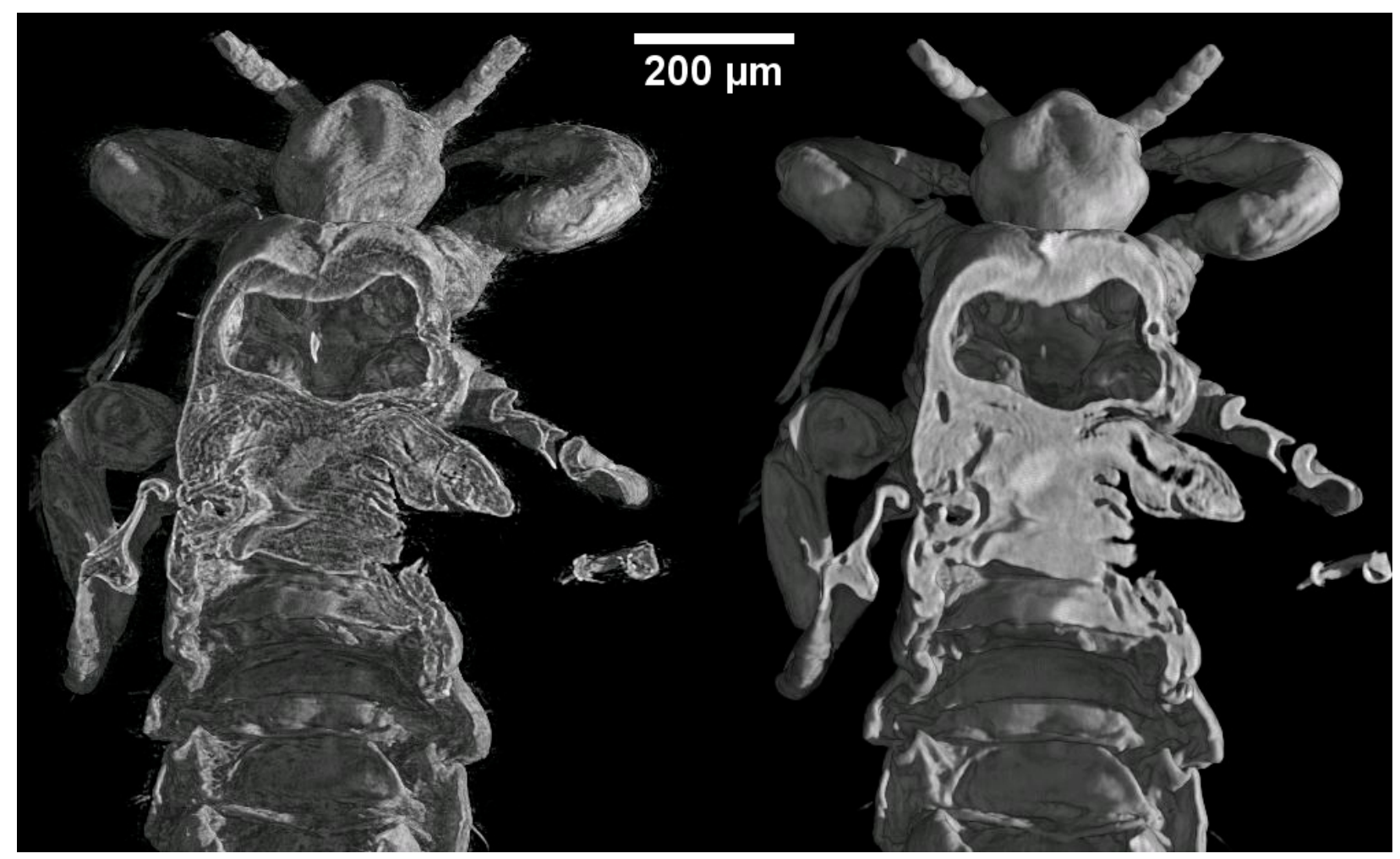

Figure 3: Rendering of a head louse. Phase contrast artefacts disturb the FBP reconstruction (left), while MBA-reconstructed volume is smooth but has a loss of detail (right)
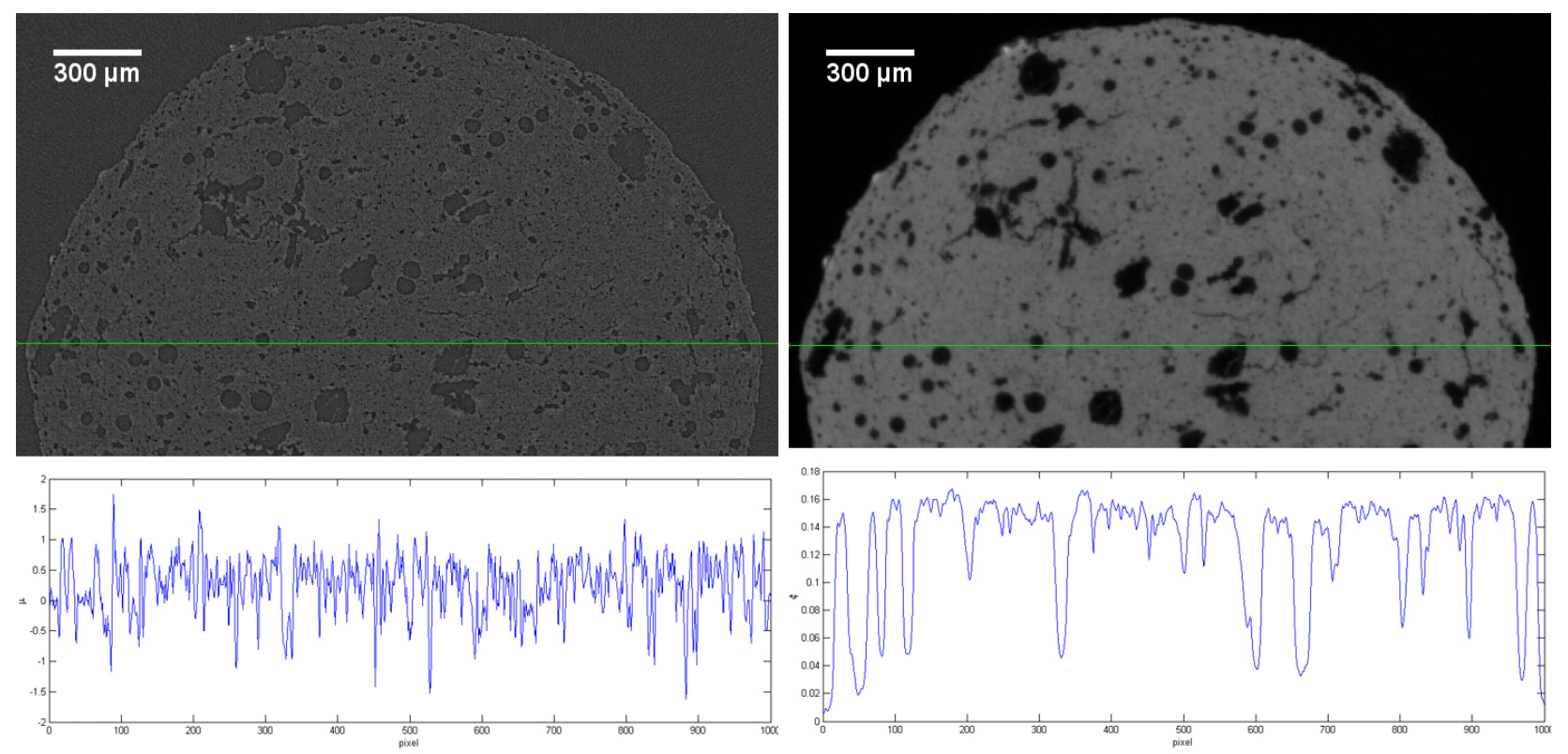

Figure 4: Reconstructed slice from a pharmaceutical sample. The noise and artefacts present in the FBP reconstruction (left) are removed, but some details are lost in the MBA reconstruction (right) 
[2] A. Kak, M. Slaney, Principles of Computerized Tomographic Imaging, IEEE Press, 1988.

[3] F. Pfeiffer, T. Weitkamp, O. Bunk, et al., Phase retrieval and differential phase-contrast imaging with low-brilliance X-ray sources, Nat. Phys. 2 (4) (2006) 258-261.

[4] F. Pfeiffer, O. Bunk, C. Kottler, et al., Tomographic reconstruction of three-dimensional objects from hard X-ray differential phase contrast projection images, Nucl. Instrum. Meth. Phys. Res. A 580 (2) (2007) 925-928.

[5] E. Pagot, S. Fiedler, P. Cloetens, et al., Quantitative comparison between two phase contrast techniques: diffraction enhanced imaging and phase propagation imaging, Phys. Med. Biol. 50 (2005) 709-724.

[6] S. Wilkins, T. Gureyev, D. Gao, et al., Phase-contrast imaging using polychromatic hard X-rays, Nature 384 (1996) 335-338.

[7] M. Born, E. Wolf, Principles of Optics, Pergamon, Oxford, 1980.

[8] M. Teague, Deterministic phase retrieval: a Green's function solution, J. Opt. Soc. Am. 73 (11) (1983) 1434-1441.

[9] A. Peterzol, A. Olivo, L. Rigon, et al., The effects of the imaging system on the validity limits of the ray-optical approach to phase contrast imaging, Med. Phys. 32 (12) (2005) 3617-3627.

[10] M. Langer, P. Cloetens, J. Guigay, et al., Quantitative comparison of direct phase retrieval algorithms in in-line phase tomography, Med. Phys. 35 (10) (2008) 4556-4566.

[11] K. Nugent, T. Gureyev, D. Cookson, et al., Quantitative Phase Imaging Using Hard X Rays, Phys. Rev. Lett. 77 (14) (1996) 2961-2964.

[12] X. Wu, H. Liu, A general theoretical formalism for X-ray phase contrast imaging, J. X-ray Sci. Tech. 11 (2003) 33-42.

[13] A. Bronnikov, Reconstruction formulas in phase-contrast tomography, Opt. Comm. 171 (1999) 239-244.

[14] A. Bronnikov, Phase-contrast CT: Fundamental theorem ans fast image reconstruction algorithms, Proc. SPIE 6318 (2006) $27-33$.

[15] A. Groso, R. Abela, M. Stampanoni, Implementation of a fast method for high resolution phase contrast tomography, Opt. Express 14 (18) (2006) 8103-8110.

[16] D. Paganin, S. Mayo, T. Gureyev, et al., Simultaneous phase and amplitude extraction from a single defocused image of a homogeneous object, J. Microscopy 206 (2002) 33-40.

[17] S. Mayo, T. Davis, T. Gureyev, et al., X-ray phase-contrast microscopy and microtomography, J. Opt. Soc. Am. 11 (19) (Opt. Express) 2289-2302

[18] B. Masschaele, V. Cnudde, M. Dierick, et al., UGCT: New xray radiography and tomography facility, Nucl. Instrum. Meth. Phys. Res. A 580 (1) (2007) 266-269.

[19] J. Vlassenbroeck, M. Dierick, B. Masschaele, et al., Software tools for quantification of X-ray microtomography, Nucl. Instrum. Meth. Phys. Res. A 580 (1) (2007) 442-445. 